\title{
Comparação das estimativas de prevalência de indicadores de saúde no Município de Campinas, São Paulo, Brasil, nos anos de 2001/2002 (ISA-SP) e 2008/2009 (ISA-Camp)
}

\section{Comparison of prevalence estimates for health indicators in Campinas, São Paulo State, Brazil, in 2001-2002 (ISA-SP) and 2008-2009 \\ (ISA-Camp)}

\section{Comparación de las estimaciones de prevalencia en los indicadores de salud en Campinas, São Paulo, Brasil, durante los años 2001/2002 (ISA-SP) y $2008 / 2009$ (ISA-Camp)}

Priscila Maria Stolses Bergamo Francisco ${ }^{1}$ Marilisa Berti de Azevedo Barros ${ }^{1}$ Neuber José Segri 2 Maria Cecília Goi Porto Alves 3 Chester Luis Galvão César 2 Luana Carandina ${ }^{4}$ Moisés Goldbaun 5

\footnotetext{
${ }_{1}^{1}$ Faculdade de Ciências Médicas, Universidade Estadual de Campinas, Campinas, Brasil.

2 Faculdade de Saúde Pública, Universidade de São Paulo, São Paulo, Brasil. 3 Instituto de Saúde. Secretaria de Estado da Saúde, São Paulo, Brasil. ${ }_{4}^{4}$ Faculdade de Medicina de Botucatu, Universidade Estadual Paulista Júlio de Mesquita Filho, Botucatu, Brasil.

5 Faculdade de Medicina Universidade de São Paulo, São Paulo, Brasil.

Correspondência P. M. S. B. Francisco Faculdade de Ciências Médicas, Universidade Estadual de Campinas. Rua Tessália Vieira de Camargo, 126, Campinas, $S P$ 13083-887, Brasil. primaria@fcm.unicamp.br
}

\begin{abstract}
The aim of the study was to compare prevalence estimates of health indicators for adults living in Campinas, São Paulo State, using data from two household surveys (ISA-SP 2001-2002 and ISA-Camp 2008-2009), analyzing data from 941 and 2,637 individuals 18 years and older, respectively. Socio-demographic variables were used to characterize the study population. Prevalence rates and $95 \%$ confidence intervals were estimated, and comparisons were performed by prevalence ratios adjusted for sex, age, and education, obtained by Poisson regression with robust variance. Statistically significant differences were observed for prevalence of morbidity, medication, smoking, and lifetime Pap smear and mammogram. Surveillance of health indicators by repeated surveys in the same population can facilitate monitoring goals and objectives by providing support to plan public health interventions.
\end{abstract}

Health Surveys; Health Status Indicators; Population Surveys

\section{Resumo}

O objetivo do estudo foi comparar estimativas da prevalência de indicadores de saúde para adultos residentes em Campinas, São Paulo, Brasil, utilizando dados de inquéritos domiciliares realizados em diferentes períodos de tempo (ISA-SP 2001/2002 e ISA-Camp 2008/2009), com amostras de 941 e 2.637 indivíduos de 18 anos e mais, respectivamente. Variáveis sociodemográficas caracterizaram a população estudada. Foram estimadas prevalências e seus respectivos intervalos de 95\% de confiança e as comparações foram realizadas pelas razões de prevalência ajustadas por sexo, idade e escolaridade, obtidas pela regressão de Poisson com variância robusta. Diferenças estatisticamente significantes foram observadas para as prevalências de: morbidade referida, uso de medicamentos, percentual dos que nunca fumaram, realização dos exames de Papanicolaou e de mamografia, alguma vez na vida. O acompanhamento de indicadores de saúde por inquéritos repetidos em uma mesma população pode facilitar o monitoramento de objetivos e metas fornecendo subsídios ao planejamento de ações em saúde.

Inquéritos Epidemiológicos; Indicadores Básicos de Saúde; Inquéritos Demográficos 


\section{Introdução}

Conhecer o estado de saúde de uma população por meio de informações apoiadas em dados válidos e confiáveis é condição fundamental para a análise objetiva da situação de saúde, para a definição de prioridades e programação de ações, para a avaliação do impacto de intervenções e para auxiliar o planejamento e a gestão em saúde $1,2,3$.

Os inquéritos de saúde de base populacional são ferramentas amplamente reconhecidas para o levantamento de tais informações e possibilitam, ainda, descrever e monitorar o grau de disparidades entre subgrupos 4,5,6, analisar diferenciais da concentração de renda na saúde 7 e das intervenções sob diferentes aspectos 5,8 .

O monitoramento da tendência do estado de saúde, dos comportamentos relacionados à saúde, do acesso e uso dos serviços pela população, requer a utilização de inquéritos periódicos de saúde 1,9,10,11,12, adequados para identificar mudanças temporais nos fatores de risco e na frequência de doenças crônicas nas populações 12,13. Essa estratégia de pesquisa tem sido utilizada internacionalmente há algumas décadas 14 e mais recentemente também no Brasil 2,13, com o objetivo de coletar informações para avaliar as várias dimensões da saúde da população, permitindo o acompanhamento das flutuações e tendências históricas do padrão epidemiológico de diferentes coletividades consideradas à mesma época, ou da mesma população em períodos de tempo distintos.

$\mathrm{Na}$ área da saúde, experiências nacionais em relação à comparação de dados de inquéritos periódicos ainda são recentes. A Pesquisa Nacional por Amostras de Domicílio (PNAD) do Instituto Brasileiro de Geografia e Estatística (IBGE), inquérito de base populacional com abrangência nacional realizado a partir de amostra probabilística da população incluiu o primeiro suplemento de saúde em 1981. Desde 1998, os suplementos vêm sendo aplicados sistematicamente, a cada cinco anos, possibilitando o acompanhamento das tendências de vários indicadores $2,4,7$. A abrangência temática dessa pesquisa possibilitou a realização de inúmeros estudos sobre condições de saúde e uso de serviços de saúde 2 .

Mais recentemente, no ano de 2006, o sistema VIGITEL - Vigilância de Fatores de Risco e Proteção para Doenças Crônicas por Inquérito Telefônico, foi implantado no país, objetivando monitorar continuamente a frequência e distribuição de fatores de risco e proteção para doenças crônicas nas capitais de todos os estados brasileiros e no Distrito Federal, por meio da realização de entrevistas telefônicas assistidas por computador em amostras probabilísticas da população adulta residente em domicílios servidos por linhas de telefone fixo residencial 15 .

A atualização periódica de informações e comparações sequenciais em áreas geográficas delimitadas provenientes de levantamentos realizados na mesma população em períodos de tempo distintos, aplicando-se técnicas já desenvolvidas para tais análises 16 se justifica, considerando-se que o acompanhamento de indicadores de saúde por inquéritos repetidos, pode facilitar o monitoramento de objetivos e metas em saúde, além de fornecer subsídios ao planejamento de ações em saúde.

O objetivo do presente estudo foi comparar estimativas da prevalência de variáveis de interesse para a saúde, utilizando dados de inquéritos domiciliares realizados em diferentes períodos de tempo no Município de Campinas, São Paulo, Brasil (ISA-SP 2001/2002 e ISA-Camp 2008/2009).

\section{Métodos}

Neste estudo foram utilizados dados da população adulta de 18 anos e mais do ISA-SP (Inquérito de Saúde no Estado de São Paulo) e do ISA-Camp (Inquérito de Saúde no Município de Campinas), inquéritos domiciliares de saúde de base populacional realizados no município, nos anos de 2001/2002 e 2008/2009, respectivamente.

\section{ISA-SP}

O ISA-SP foi uma pesquisa desenhada para analisar condições de vida, situação de saúde e uso de serviços de saúde em diferentes áreas do Estado de São Paulo (Campinas, Distrito do Butantã, Região Sudoeste da Grande São Paulo e Botucatu) e cuja coleta de dados foi realizada por entrevistas domiciliares entre abril de 2001 e março de 200217.

Para garantir números mínimos na amostra para análises de cada uma das áreas de estudo, foram definidos grupos de idade e sexo, a saber: população menor de um ano; população de 1-11 anos; homens de 12-19 anos; mulheres de 12-19 anos; homens de 20-59 anos; mulheres de 20-59 anos; homens de 60 anos ou mais e mulheres de 60 anos ou mais 17 .

A amostra foi obtida por procedimentos de amostragem probabilística, estratificada, por conglomerados e em dois estágios: setor censitário e domicílio. Com base nos dados da contagem da população realizada pelo IBGE em 1996, os setores censitários foram classificados em três estratos, segundo o percentual de chefes de famí- 
lia com nível universitário: menos de 5\%, de 5 a $24,99 \%$ e com $25 \%$ ou mais, visando aumentar a probabilidade de inclusão de indivíduos pertencentes ao estrato de maior nível socioeconômico da população, proporcionando análise da situação de saúde e uso de serviços de saúde segundo subgrupos sociais. De cada estrato, foram sorteados 10 setores censitários com probabilidade proporcional ao tamanho, expresso pelo número de domicílios, por meio de sorteio sistemático, aplicado à relação de unidades primárias, ordenadas segundo tipo de setor. Após arrolamento para atualização de mapas e contagem dos domicílios, foram selecionados os que compuseram a amostra de cada área e estrato. Em cada domicílio, foram entrevistados os indivíduos segundo domínios de sexo e idade previamente definidos 18 .

O número de pessoas para compor a amostra foi obtido considerando-se a situação correspondente à máxima variabilidade para a frequência dos eventos estudados ( $p=0,50)$; um coeficiente de 95\% de confiança na determinação dos intervalos de confiança $(z=1,96)$; um erro de amostragem de $10 \%$ e efeito de delineamento igual a 218 .

Adicionalmente, considerou-se um percentual de $20 \%$ de perdas para corrigir os efeitos de não resposta (casa fechada e recusa), o que elevou de 200 para 250 o tamanho total da amostra em cada domínio do estudo. Com isso, no Município de Campinas foram entrevistadas 1.585 pessoas de todas as idades e, para cada uma delas foi calculado um peso total, resultado da multiplicação do peso do delineamento e do peso do ajuste de pós-estratificação, considerando a distribuição por sexo e idade (domínios), segundo o Censo Demográfico de 200018.

\section{ISA-Camp}

Com o objetivo de analisar os perfis do estado de saúde, estilo de vida (fatores de risco de doenças crônicas) e uso de serviços de saúde de diferentes segmentos sociais da população, avaliando o grau de equidade/desigualdade social, o Centro Colaborador em Análise de Situação de Saúde (CCAS), Faculdade de Ciências Médicas, Universidade Estadual de Campinas (Unicamp), realizou o ISA-Camp.

Os dados foram levantados por entrevistas domiciliares na área urbana do Município de Campinas entre janeiro de 2008 e abril de 2009. Foram coletadas informações da população residente não institucionalizada com 10 anos ou mais de idade.

Selecionou-se uma amostra probabilística, por conglomerados em dois estágios: setores censitários e domicílios. No primeiro estágio, foram sorteados 50 setores censitários com probabilidade proporcional ao tamanho (número de domicílios), por meio de sorteio sistemático, ordenando-se os setores pelo percentual de chefes de família que possuíam nível universitário. No segundo, foram sorteados os domicílios dos setores selecionados. O tamanho da amostra foi calculado considerando-se a situação correspondente à máxima variabilidade para a frequência dos eventos estudados ( $p=0,50$ ), nível de 95\% de confiança na determinação dos intervalos de confiança $(z=1,96)$, erro de amostragem entre 4 e 5 pontos percentuais e efeito de delineamento igual a 2, totalizando mil indivíduos em cada domínio de idade pré-definido: adolescentes (10-19 anos), adultos (20-59 anos) e idosos (60 anos e mais). Esperando-se uma taxa de $80 \%$ de resposta, o tamanho da amostra foi corrigido para 1.250 pessoas. Em cada domicílio foram coletadas informações de todos os moradores da faixa etária selecionada para aquele domicílio. O peso final atribuído a cada indivíduo entrevistado foi o resultado da multiplicação do peso do delineamento, do peso da não resposta e do peso da pós-estratificação, considerando-se a distribuição segundo sexo (masculino e feminino) e faixas etárias (10-19, 20-39, 40-59, 60 anos e mais de idade) da Fundação Sistema Estadual de Análise de Dados (Fundação Seade, 2007; plano de amostragem disponível em: http://www.fcm. unicamp.br/centros/ccas/arquivos/plano_de_ amostragem.pdf, acessado em 20/Jun/2012).

Neste estudo foram analisados dados de 941 e 2.637 indivíduos de 18 anos ou mais residentes em Campinas, entrevistados pelo ISA-SP e ISACamp, respectivamente. A partir da revisão do conteúdo dos instrumentos de medida utilizados em ambas as pesquisas, considerando a importância epidemiológica e a compatibilização dos dois bancos de dados utilizados, foram selecionadas para o presente estudo:

Variáveis sociodemográficas: sexo, idade, escolaridade (em anos de estudo), cor da pele/raça e situação conjugal.

Variáveis de saúde: morbidade referida nos 15 dias que antecederam a entrevista, a partir da pergunta: "Você teve algum problema de saúde nas últimas 2 semanas?” (sim ou não), presença de hipertensão arterial, diabetes, doença do coração, artrite/reumatismo/artrose, osteoporose, asma/bronquite/enfisema, uso de medicamentos investigado pela questão: "Você usou algum medicamento nos últimos 3 dias?" (sim ou não), presença de doenças crônicas (uma, duas ou mais), autoavaliação de saúde ruim ou muito ruim entre idosos, excesso de peso e obesidade, calculados com dados de peso e altura referidos 
e pontos de corte propostos pela Organização Mundial da Saúde $19\left(25 \leq \mathrm{IMC}<30 \mathrm{~kg} / \mathrm{m}^{2}\right.$ e IMC $\geq$ $30 \mathrm{~kg} / \mathrm{m}^{2}$, respectivamente), prática de atividade física no contexto de lazer (pratica e não pratica), investigada pela pergunta: "Você pratica regularmente, pelo menos uma vez por semana algum tipo de exercício físico ou esporte?", tabagismo (percentual dos que fumam atualmente e dos que nunca fumaram), internação hospitalar nos últimos 12 meses, realização de consulta odontológica no ano prévio, realização de Papanicolaou em mulheres de 20 anos e mais e de mamografia em mulheres de 40 anos e mais alguma vez na vida, e cobertura vacinal contra gripe em idosos (nos últimos 12 meses).

As variáveis sexo, idade, escolaridade (em anos de estudo), cor da pele/raça e situação conjugal foram utilizadas para caracterizar a população estudada por ambos os inquéritos. As análises foram realizadas a partir de um único arquivo de dados combinado de registros com informações para todas as variáveis estudadas. As variáveis foram renomeadas e, em seguida, classificadas com valores e rótulos idênticos para a mesma categoria de resposta e uma nova variável foi criada para identificar a origem da informação (ISA-SP ou ISA-Camp), possibilitando as comparações 16. Ainda, na análise dos dados, considerando-se os diferenciais no delineamento de ambos os inquéritos, além da unidade primária de amostragem, ou seja, dos setores censitários, os estratos foram definidos por meio de uma variável categórica contendo valores de 1 a 4 . O valor " 4 " foi considerado para todos os registros relativos ao ISA-Camp e os demais valores (1, 2 e 3) referiram-se aos três estratos da pesquisa ISASP. Assim, como no ISA-Camp a amostra não foi estratificada, os dados foram analisados como se houvesse um único estrato, não comprometendo a comparabilidade dos resultados, que foram também averiguados pelo cálculo das prevalências e seus respectivos intervalos de confiança, estimados a partir do banco de dados de cada um dos inquéritos, separadamente.

Para os indicadores selecionados neste estudo foram estimadas as prevalências e seus respectivos intervalos de $95 \%$ de confiança (IC95\%). As razões de prevalência usadas nas comparações para dimensionar a magnitude das diferenças entre as estimativas obtidas pelos inquéritos foram calculadas pela regressão de Poisson com variância robusta. Para controlar possíveis diferenças nas distribuições amostrais das pesquisas, devidas aos diferentes pesos de pós-estratificação, calculados com base em anos distintos (ISA-SP - Censo Demográfico, IBGE 2000; e ISA-Camp - Fundação Seade, 2007), sexo, idade e escolaridade foram usadas como variá- veis de ajuste nos modelos de regressão. Considerou-se a característica estudada como variável dependente e a variável criada para identificar a origem do inquérito (ISA-SP ou ISA-Camp) como independente. Deste modo, segundo Lee et al. 16, se a variável independente apresenta efeito estatisticamente significativo, existem evidências para rejeitar a hipótese de que as estimativas obtidas pelos dois inquéritos sejam similares, com um nível descritivo de 0,05 para o teste de Wald. Na análise dos dados, foram usados os comandos do grupo svy do programa Stata 11.0 (Stata Corp., College Station, Estados Unidos), cujos procedimentos para análise de inquéritos populacionais incorporam as ponderações necessárias, produzindo melhor estimativa do erro padrão, o qual é influenciado pela conglomeração das unidades e estratificação derivadas do delineamento amostral complexo 20,21.

Em ambas as pesquisas, os objetivos foram apresentados aos indivíduos sorteados solicitando-se, após conhecimento do tipo de informação que seria requerida, o consentimento assinado de participação. O projeto de pesquisa do ISA-SP foi apresentado e aprovado pelas Comissões de Ética das três universidades participantes: Universidade de São Paulo (USP), Unicamp (parecer no 369/2000) e Universidade Estadual Paulista Júlio de Mesquita Filho (UNESP). O projeto para realização do ISA-Camp, foi aprovado pela Comissão de Ética da Unicamp de Campinas (parecer ㄲo 079/2007).

\section{Resultados}

O perfil sociodemográfico da população estudada pode ser observado na Tabela 1 . Na distribuição segundo sexo e idade, observam-se semelhanças com predomínio de mulheres e adultos na faixa etária de 30-59 anos. Verifica-se, ainda, maior proporção de adultos com 8 ou mais anos de estudo, de cor de pele/raça branca e dos que vivem com cônjuge para ambos os períodos analisados.

Na Tabela 2 são apresentadas as estimativas da prevalência segundo indicadores de saúde selecionados. No que se refere aos eventos relacionados à saúde, foram verificadas diferenças nas estimativas obtidas para a presença de morbidade nos últimos 15 dias, com maior prevalência observada em 2008/2009 (RP = 1,31; IC95\%: 1,031,68). Quanto ao uso de medicamentos nos três dias que antecederam à pesquisa, também houve uma elevação significativa da prevalência no período ( $\mathrm{RP}=1,14$; IC95\%: 1,02-1,28). Na avaliação dos indicadores de hábitos e comportamentos, observou-se aumento significativo do percentual 
Distribuição percentual da população adulta (18 anos ou mais), segundo características sociodemográficas e ano de realização dos inquéritos. ISA-SP (2001/2002) e ISA-Camp (2008/2009). Campinas, São Paulo, Brasil.

\begin{tabular}{|c|c|c|c|c|}
\hline \multirow{2}{*}{$\begin{array}{l}\text { Características } \\
\text { sociodemográficas }\end{array}$} & \multicolumn{2}{|c|}{ ISA-SP } & \multicolumn{2}{|c|}{ ISA-Camp } \\
\hline & $n$ * & $\%(I C 95 \%) * *$ & $n$ * & $\%(I C 95 \%) * *$ \\
\hline \multicolumn{5}{|l|}{ Sexo } \\
\hline Masculino & 481 & $47,8(43,5-52,1)$ & 1.141 & $47,7(45,4-50,1)$ \\
\hline Feminino & 460 & $52,2(47,9-56,4)$ & 1.496 & $52,3(49,9-54,6)$ \\
\hline \multicolumn{5}{|l|}{ Faixa etária (anos) } \\
\hline $18-29$ & 242 & $31,5(27,6-35,6)$ & 466 & $29,8(26,9-32,8)$ \\
\hline $30-59$ & 273 & $54,5(50,5-58,5)$ & 652 & $54,7(51,7-57,6)$ \\
\hline 60 e mais & 426 & $14,0(12,0-16,2)$ & 1.519 & $15,5(13,5-17,8)$ \\
\hline \multicolumn{5}{|l|}{ Escolaridade (anos) } \\
\hline $0-3$ & 208 & $14,9(12,2-18,1)$ & 613 & $10,6(8,5-13,2)$ \\
\hline $4-7$ & 278 & $25,9(22,6-29,5)$ & 739 & $22,7(18,9-26,9)$ \\
\hline $8-11$ & 325 & $40,1(35,2-45,1)$ & 881 & $44,2(39,6-48,8)$ \\
\hline 12 ou mais & 127 & $19,1(15,3-23,6)$ & 398 & $22,5(15,4-31,7)$ \\
\hline \multicolumn{5}{|l|}{ Raça/Cor da pele } \\
\hline Branca & 763 & $79,9(75,8-83,5)$ & 1.961 & $73,6(67,8-78,7)$ \\
\hline Não-branca & 176 & $20,1(16,5-24,2)$ & 672 & $26,3(21,3-32,2)$ \\
\hline \multicolumn{5}{|l|}{ Situação conjugal } \\
\hline Com cônjuge & 523 & $58,2(53,8-62,5)$ & 1.453 & $59,1(55,4-62,7)$ \\
\hline Sem cônjuge & 417 & $41,8(37,5-46,2)$ & 1.184 & $40,9(37,3-44,6)$ \\
\hline
\end{tabular}

* Número de indivíduos na amostra não ponderada;

** Prevalências e intervalos de 95\% de confiança (IC95\%) calculados sob ponderações devidas ao desenho amostral.

de pessoas que nunca fumaram no período $(\mathrm{RP}=$ 1,14; IC95\%: 1,03-1,26) (Tabela 2).

Para o uso e acesso a serviços de saúde, também apresentados na Tabela 2, as estimativas da prevalência de mulheres que realizaram Papanicolaou e mamografia alguma vez na vida, apresentaram diferenças estatisticamente significativas. Particularmente em relação ao Papanicolaou, os dados do ISA-Camp revelaram que a prevalência de realização entre as mulheres de 20 anos ou mais em 2008/2009 foi maior em relação ao período de 2001/2002 ( $\mathrm{RP}=1,07$; IC95\%: 1,01 1,13). Em 2008/2009, por meio do ISA-Camp, a prevalência da realização de mamografia para as mulheres com 40 anos ou mais foi de $80,0 \%$ (IC95\%: 75,5-84,5), enquanto em 2001/2002, pelo inquérito ISA-SP, a prevalência estimada foi de apenas 57,7\% (IC95\%: 48,9-66,6) denotando, após ajuste por idade e escolaridade, uma elevação de cerca de $36 \%$ na cobertura do referido exame preventivo em Campinas $(\mathrm{RP}=1,36$; IC95\%: 1,16-1,60).

\section{Discussão}

Informações de inquéritos populacionais sobre a prevalência de fatores de risco modificáveis permitem o monitoramento e a avaliação de alterações nos perfis de morbidade e mortalidade ao longo do tempo 9,10,11,12. Este estudo comparou estimativas de indicadores comumente investigados em inquéritos de saúde, obtidas por entrevistas domiciliares realizadas no mesmo município. Os inquéritos apresentaram resultados semelhantes para a maioria dos indicadores examinados, denotando estabilidade no período, exceto para morbidade referida nos últimos 15 dias, uso de medicamentos nos três dias que antecederam as pesquisas, percentual de não fumantes, realização de Papanicolaou e de mamografia na vida, cujas estimativas apresentaram diferenças de magnitudes diversas e significativamente maiores pelo ISA-Camp (2008/2009).

Entre os indicadores de saúde avaliados neste estudo, alguns se destacam pela importância epidemiológica que vêm apresentando ao longo do tempo. Constituem fatores que associados ao aumento do risco de se desenvolver uma doença, passaram a ser denominados fatores de risco. A 
Prevalências e razões de prevalências ajustadas dos indicadores de saúde selecionados da população adulta (18 anos ou mais), segundo ano de realização dos inquéritos. ISA-SP (2001/2002) e ISA-Camp (2008/2009). Campinas, São Paulo, Brasil.

\begin{tabular}{|c|c|c|c|c|c|c|}
\hline \multirow[t]{2}{*}{ Desfechos binários de saúde } & \multicolumn{2}{|c|}{$\begin{array}{c}\text { ISA-SP } \\
(n=941)\end{array}$} & \multicolumn{2}{|c|}{$\begin{array}{l}\text { ISACamp } \\
(n=2.637)\end{array}$} & \multirow[t]{2}{*}{$\mathbf{R P}_{\text {ajustada }}$} & \multirow[t]{2}{*}{ Valor de $\mathrm{p}$} \\
\hline & $\%$ & IC95\% & $\%$ & IC $95 \%$ & & \\
\hline \multicolumn{7}{|l|}{ Eventos relacionados à saúde } \\
\hline Presença de morbidade nos últimos 15 dias & 15,1 & $12,0-18,1$ & 19,7 & $17,0-22,4$ & $1,31(1,03-1,69)$ & 0,031 \\
\hline Hipertensão arterial & 20,9 & $16,8-24,9$ & 19,8 & $17,8-21,7$ & $0,92(0,77-1,09)$ & 0,336 \\
\hline Diabetes & 4,4 & $2,9-6,0$ & 6,4 & $5,1-7,8$ & $1,38(0,93-2,05)$ & 0,107 \\
\hline Doença do coração & 6,0 & $3,9-8,1$ & 5,7 & $4,5-7,0$ & $0,91(0,62-1,35)$ & 0,648 \\
\hline Reumatismo/Artrite/Artrose & 7,4 & $5,6-9,3$ & 6,9 & $5,6-8,3$ & $0,87(0,67-1,13)$ & 0,298 \\
\hline Osteoporose & 2,9 & $1,8-3,9$ & 3,1 & $2,3-3,8$ & $0,98(0,66-1,44)$ & 0,901 \\
\hline Asma/Bronquite/Enfisema & 4,1 & $2,6-5,6$ & 3,9 & $2,8-5,1$ & $0,97(0,60-1,56)$ & 0,904 \\
\hline Uso de medicamentos nos 3 dias anteriores à pesquisa & 48,5 & $43,3-53,7$ & 56,4 & $53,1-59,8$ & $1,14(1,02-1,28)$ & 0,021 \\
\hline \multicolumn{7}{|l|}{ Número de doenças crônicas referidas * } \\
\hline 1 & 18,4 & $15,1-21,7$ & 19,0 & $17,0-20,9$ & $1,01(0,82-1,24)$ & 0,908 \\
\hline 2 ou mais & 11,4 & $9,1-13,6$ & 10,8 & $9,2-12,5$ & $0,91(0,73-1,12)$ & 0,360 \\
\hline Autoavaliação de saúde ruim/muito ruim em idosos & 10,4 & $7,3-13,6$ & 12,1 & $10,0-14,3$ & $1,17(0,83-1,66)$ & 0,360 \\
\hline \multicolumn{7}{|l|}{ Índice de massa corporal } \\
\hline Excesso de peso & 44,7 & $40,3-49,2$ & 47,1 & $44,1-50,1$ & $1,04(0,93-1,17)$ & 0,455 \\
\hline Obesidade & 14,0 & $11,0-16,9$ & 15,5 & $12,8-18,2$ & $1,09(0,83-1,44)$ & 0,508 \\
\hline \multicolumn{7}{|l|}{ Hábitos e comportamentos } \\
\hline Prática de atividade física no lazer & 33,8 & $30,6-37,0$ & 33,8 & $28,8-38,8$ & $0,95(0,81-1,11)$ & 0,516 \\
\hline Fuma cigarros atualmente & 22,7 & $18,2-27,2$ & 18,8 & $16,1-21,4$ & $0,87(0,69-1,09)$ & 0,222 \\
\hline Nunca fumou & 59,4 & $54,4-64,4$ & 68,6 & $64,20-72,9$ & $1,14(1,03-1,26)$ & 0,013 \\
\hline \multicolumn{7}{|l|}{ Uso/Acesso a serviços de saúde } \\
\hline Internação hospitalar no último ano & 10,5 & $8,2-12,8$ & 9,8 & $8,3-11,3$ & $0,92(0,71-1,20)$ & 0,556 \\
\hline Consulta odontológica no ano prévio & 55,3 & $50,7-59,9$ & 53,8 & $47,5-60,0$ & $0,93(0,83-1,04)$ & 0,217 \\
\hline Exame de Papanicolaou ** & 86,5 & $81,8-91,2$ & 92,8 & $90,8-94,8$ & $1,07(1,01-1,13)$ & 0,023 \\
\hline Exame de mamografia ** & 57,7 & $48,9-66,6$ & 80,0 & $75,5-84,5$ & $1,36(1,16-1,60)$ & $<0,001$ \\
\hline Vacina contra gripe em idosos & 65,9 & $60,4-71,4$ & 62,6 & $59,4-65,8$ & $0,95(0,86-1,05)$ & 0,352 \\
\hline
\end{tabular}

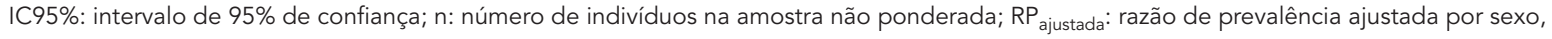

idade e escolaridade.

* Hipertensão, diabetes, doença cardiovascular, câncer (tumor maligno), artrite/reumatismo/artrose, osteoporose, asma/bronquite/enfisema;

** Razão de prevalência ajustada por idade e escolaridade (categoria de referência: ISA-SP 2001/2002).

hipertensão arterial (doença crônica considerada fator de risco para acometimento vascular), o excesso de peso, o sedentarismo e o tabagismo, além do consumo excessivo de bebidas alcoólicas, estão entre os mais estudados na atualidade e, isolados ou combinados, têm permitido estabelecer associações entre eles e muitas doenças, principalmente as cardiovasculares 11,22 e alguns tipos de câncer.

Nos inquéritos de saúde de base populacional, é comum o emprego de indicadores de morbidade referida pelo próprio entrevistado. Tais indicadores são averiguados por questões globais (informação simples sobre a ocorrência ou não de sintomas ou doenças - sim ou não) ou específicas (estimuladas por um checklist de doenças ou problemas de saúde). As estimativas obtidas a partir das duas formas de informação podem ser diferentes e variar segundo gênero 23 , no entanto, a morbidade referida constitui a única forma de avaliação do perfil de morbidade em grupos populacionais. Neste estudo, foi possível observar aumento da prevalência de morbidade referida nos 15 dias que antecederam a pesquisa pela população adulta de Campinas. Destaca-se que, para as doenças específicas investigadas não houve alteração no período, bem como para a presença de doenças crônicas, o que indicaria 
um aumento na percepção do adoecimento pela população.

O uso crescente de medicamentos tem sido preocupação constante entre os profissionais e gestores da área de saúde pois, ao mesmo tempo que faz parte do processo de cura, também pode trazer riscos à saúde quando não utilizados por meio de uma farmacoterapia adequada 24 . Neste estudo, observou-se aumento significativo da prevalência do uso de ao menos um medicamento nos três dias que antecederam a pesquisa em Campinas, o que pode ser parcialmente explicado por sua forte associação com a morbidade referida nos últimos 15 dias em ambos os inquéritos, revelando seu uso como a principal intervenção terapêutica empregada tanto pelas pessoas com morbidades agudas quanto pelas portadoras de doenças crônicas ${ }^{24}$. Deve-se considerar que este estudo utilizou dados de inquéritos de saúde com temática abrangente, o que pode levar a resultados diferentes daqueles que seriam obtidos numa pesquisa específica sobre $o$ tema, entretanto, verifica-se coerência dos achados com resultados anteriores 25 .

Diferentes instrumentos podem ser utilizados para apreender a percepção do estado de saúde da população. A autoavaliação da saúde, considerada um importante indicador subjetivo do nível individual e coletivo de saúde e obtida por uma única questão, tem sido muito utilizada nos inquéritos de saúde 26,27. Associada a medidas objetivas de morbidade e de uso de serviços, a pior saúde percebida torna-se um poderoso preditor de mortalidade e do declínio funcional nos idosos 28,29,30, independentemente de outros fatores, além de ser equivalente a indicadores obtidos por instrumentos mais complexos e extensos 30. A obtenção do indicador de autoavaliação de saúde por meio de estudos de base populacional possibilita o conhecimento das condições de saúde percebidas pelos indivíduos. O aumento da prevalência da saúde autopercebida como ruim/ muito ruim indicaria a necessidade de intensificação de políticas e programas de promoção da saúde e da ampliação de cuidados para evitar ou postergar a incidência de doenças e de suas consequências 26 . No período avaliado, este indicador apresentou prevalência em torno de $10 \% \mathrm{e}$ estabilidade na população idosa de Campinas.

Recentemente, o peso e estatura autorreferidos surgiram como uma alternativa à aferição de medidas antropométricas in loco, tornando possíveis estudos epidemiológicos em grandes populações, devido à redução de custos e facilidade no trabalho de campo. A importância da obtenção desses dados reside no fato de que alterações no estado nutricional contribuem para o aumento da morbidade e mortalidade. Particu- larmente o sobrepeso e a obesidade são fatores de risco conhecidos para vários agravos à saúde, tais como isquemia cardíaca, hipertensão arterial, acidente vascular cerebral, diabetes mellitos tipo 2, osteoartrite, neoplasia maligna de mama pós-menopausa e de endométrio, esofagite de refluxo, hérnia de hiato e problemas psicológicos 31 . Neste estudo o IMC foi calculado com dados informados pelo entrevistado e a comparação das estimativas para o excesso de peso e obesidade revelou similaridades para a população adulta no período.

Nas últimas décadas, a atividade física tem sido crescentemente reconhecida pelos benefícios físicos - favorece a redução dos níveis de colesterol, o controle do diabetes, auxilia o equilíbrio da pressão arterial e melhora a capacidade respiratória - psicológicos e sociais decorrentes da sua prática 32,33 . Neste estudo foi avaliada a prevalência da prática de atividade física no contexto de lazer, ou seja, durante o tempo em que se pode dispor livremente para a prática dessas atividades que incluem exercícios e esportes, entre outras. Durante o período avaliado, a prevalência observada se manteve estável.

O relatório da OMS sobre a epidemia global de tabagismo 34 aponta o tabaco como fator de risco para seis das oito principais causas de morte no mundo. No Brasil, políticas de controle visando reduzir e prevenir o consumo de tabaco têm contribuído para a queda do tabagismo ${ }^{8}$ reduzindo a sobrecarga que doenças associadas ao seu uso representam para o sistema de saúde, já que qualquer exposição ao fumo (ativa ou passiva) provoca graves problemas de saúde 34 . Neste sentido, o conhecimento da extensão do problema é fundamental para a adoção e o monitoramento de ações efetivas no seu controle ${ }^{8}$. Neste estudo, as estimativas do percentual de adultos residentes em Campinas que se declararam fumantes à época das pesquisas apresentaram valores semelhantes. Para os que nunca fumaram, houve uma elevação estatisticamente significativa no período. Políticas de controle e redução do tabagismo aliadas a medidas educativas, preventivas e regulatórias têm contribuído para a queda do tabagismo no país 8,35,36, no entanto, essa tendência ainda não foi observada para a população de 18 anos e mais do município estudado.

A prevalência de internação hospitalar constitui informação essencial para a análise do acesso e uso de serviços de saúde em uma localidade. Apesar de influenciada por fatores socioeconômicos, epidemiológicos e demográficos, pela infraestrutura de serviços e políticas públicas assistenciais e preventivas, possibilita a análise de variações geográficas e temporais na distribuição das internações, identificando situações de de- 
sigualdade e tendências que demandem ações. Este indicador pode, ainda, contribuir para avaliar a adequação do volume de internações às necessidades da população atendida e subsidiar processos de planejamento, gestão e avaliação de políticas públicas voltadas para a assistência médico-hospitalar (Departamento de Informática do SUS. Proporção de internações hospitalares (SUS) por especialidade. http://www.ripsa. org.br $/$ fichasIDB $/$ record.php?node=F.5\&lang=pt \&version=ed5, acessado em 25/Jul/2012). Por se tratar de um evento mais raro e menos facilmente esquecido, o período recordatório comumente usado nos inquéritos domiciliares é de um ano. Em Campinas, cerca de $10 \%$ da população adulta referiu internação hospitalar em ambos os momentos avaliados.

A regularidade de consulta odontológica para revisão periódica é um indicador básico de atenção à saúde bucal 3,37, que também pode revelar o acesso da população em geral à assistência odontológica individual 37 . Trata-se de um indicador que, segundo Szwarcwald et al. 3 reflete de maneira singular, as desigualdades socioeconômicas em saúde e sua avaliação torna-se particularmente importante, para a formulação de políticas de saúde direcionadas a reduzir essas disparidades, já que o acesso aos serviços odontológicos é limitado e desigual no país 37 . No Município de Campinas mais de $50 \%$ da população referiu realização da consulta odontológica nos 12 meses anteriores à pesquisa em ambos os períodos, no entanto, esse percentual se manteve constante.

O teste de Papanicolaou é o exame de triagem empregado para detecção precoce do câncer de colo uterino e, para o rastreamento do câncer de mama, entre as práticas preventivas recomendadas, o exame radiológico das mamas (mamografia) é indicado na identificação de lesões subclínicas 38. Estimativas da cobertura de Papanicolaou e mamografia vêm sendo obtidas por inquéritos populacionais no país $3,39,40,41$, possibilitando o monitoramento de ações para a detecção. Este estudo comparou estimativas da cobertura de Papanicolaou em mulheres a partir de 20 anos de idade, e de mamografia em mulheres com 40 anos ou mais e verificou, em cerca de seis anos, uma elevação estatisticamente significativa das coberturas de ambos os exames, independente da escolaridade e da idade.

Em relação à avaliação da cobertura vacinal, inquéritos domiciliares realizados periodicamente permitem estimar a real proporção de pessoas vacinadas, além de possibilitar a mensuração das desigualdades sociais em relação à imunização 5 . Ainda, para Moraes \& Ribeiro 5 (p. 115), a cobertura vacinal é "um indicador de acesso ao Programa
Nacional de Imunização, podendo ser indicativo de efetividade do programa de imunização". Em Campinas, os dados da cobertura de vacinação contra a gripe na população idosa, revelaram similaridade nos períodos avaliados. Mesmo diante dos benefícios da vacinação contra influenza entre idosos e portadores de condições crônicas evidenciados em vários estudos 42,43 , da recomendação formal para vacinação nestes grupos 44, e da disponibilidade gratuita nos serviços, a adesão a esta prática preventiva ainda tem se mostrado insatisfatória no município, situação esta que pode ser modificada intensificando-se a recomendação médica ou de um profissional de saúde para ampliar as coberturas vacinais 45,46 .

O ISA-SP e o ISA-Camp são inquéritos populacionais com delineamentos amostrais semelhantes, exceto pelo fato de que no primeiro a amostra foi estratificada. Tais pesquisas utilizaram entrevistas face a face como estratégia para a coleta de dados e empregaram questões idênticas para os indicadores selecionados no presente estudo. Recentemente, estudos nacionais têm apresentado resultados inéditos no país sobre a comparação de estimativas obtidas de diferentes modalidades de inquéritos $39,40,47,48$, e de inquéritos desenvolvidos em períodos de tempo distintos 4,35.

O presente estudo estimou prevalências a partir de dados de inquéritos domiciliares realizados em diferentes períodos de tempo no $\mathrm{Mu}$ nicípio de Campinas e, em função do objetivo proposto, as variáveis originalmente politômicas (autoavaliação de saúde, índice de massa corporal, número de doenças crônicas referidas e tabagismo) foram dicotomizadas. No entanto, deve-se ressaltar que a dicotomização de variáveis dependentes policotômicas pode gerar alguma perda de informação e consequente redução do poder estatístico, dificultando a identificação da relação entre as variáveis.

O uso de informações coletadas em inquéritos com amostras complexas demanda a observação de alguns aspectos. A estimação da variância é influenciada não somente pelos pesos, mas também pela estratificação e conglomeração que podem acarretar em ganhos ou perdas de precisão nas estimativas médias 20,21. Neste estudo, a junção dos bancos de dados em um único arquivo combinado permitiu, além da utilização do módulo para análise de survey do programa Stata 11.0 que considerou os diferentes aspectos dos delineamentos amostrais complexos usados para selecionar as amostras de cada inquérito (ISA-SP e ISA-Camp), também o uso de modelos de regressão de Poisson, possibilitando o cálculo das razões de prevalência ajustadas para a comparação das estimativas. 
Inquéritos de saúde geram grande quantidade de dados, e parcerias institucionais com outros grupos de pesquisa são indispensáveis para a ampliação da capacidade de análise e utilização das informações pelos gestores e outros profissionais que atuam na área da saúde, sendo esta também uma forma de potencializar o uso dos dados coletados e reduzir custos inerentes à pesquisa ${ }^{49}$. Os inquéritos de saúde de base populacional, realizados particularmente em âmbito local, constituem uma modalidade de pesquisa relevante e necessária na formação de novos pesquisadores, que têm a oportunidade de conhecer e acompanhar todas as etapas da realização desse modelo de estudo epidemiológico.
De acordo com a OMS 50, o perfil de saúde de uma comunidade é uma descrição qualitativa e quantitativa da saúde dos indivíduos e dos fatores que a influenciam e a análise desse perfil, por meio de indicadores apropriados, possibilita a identificação de problemas e estimula ações. Neste sentido, o acompanhamento de indicadores de saúde enquanto medidas que sumarizam o efeito de determinantes distintos sobre o estado de saúde ${ }^{1}$ e que revelam a realidade das condições de saúde e uso de serviços de saúde, obtidos por inquéritos repetidos, além de permitir o conhecimento regular e sistemático de uma mesma população, pode facilitar o monitoramento de objetivos e metas provendo subsídios ao planejamento de ações em saúde.

\section{Resumen}

El objetivo del estudio fue comparar las estimaciones de prevalencia en los indicadores de salud para los adultos que viven en Campinas, São Paulo, Brasil, con datos de encuestas de diferentes períodos de tiempo (ISA-SP 2001/2002 y ISA-Camp 2008/2009), con muestras de 941 y 2.637 personas de 18 años y más, respectivamente. Las variables sociodemográficas caracterizaron a la población de estudio. Se estimaron la prevalencia y sus respectivos intervalos de confianza del 95\% y las comparaciones se realizaron por razones de prevalencia ajustadas por sexo, edad y educación, obtenidas mediante la regresión de Poisson. Se observaron dife- rencias estadísticas en la prevalencia de morbilidad, uso de medicamentos, el porcentaje de quienes nunca habían fumado, la realización de citologías vaginales y mamografías, en algún momento de sus vidas. El seguimiento de los indicadores de salud, a través de encuestas repetidas en la misma población, puede facilitar el seguimiento de las metas y objetivos y contribuir a la planificación de acciones de salud.

Encuestas Epidemiológicas; Indicadores de Salud; Encuestas Demográficas 


\section{Colaboradores}

P. M. S. B. Francisco elaborou a proposta e redação do artigo, planejou, programou e executou as análises estatísticas e realizou a revisão da literatura. M. B. A. Barros orientou a proposta do artigo, revisou as análises dos dados, contribuiu na revisão crítica e redação do texto, coordenou e desenvolveu os projetos ISA-SP e ISA-Camp. N. J. Segri colaborou nas análises estatísticas, revisão crítica e redação do texto. M. C. G. P. Alves revisou as análises estatísticas e contribuiu na revisão crítica do texto. C. L. G. César, L. Carandina e M. Goldbaum coordenaram e desenvolveram o projeto ISA-SP e colaborou na revisão do artigo.

\section{Referências}

1. Rede Interagencial de Informação para a Saúde Indicadores básicos para a saúde no Brasil: conceitos e aplicações. 2a Ed. Brasilia: Organização PanAmericana da Saúde; 2008.

2. Malta DC, Leal MC, Lima-Costa MF, Moraes Neto OL. Inquéritos nacionais de saúde: experiência acumulada e proposta para o inquérito de saúde brasileiro. Rev Bras Epidemiol 2008; 11 Suppl 1:S159-67.

3. Szwarcwald CL, Mendonça MHM, Andrade CLT. Indicadores de atenção básica em quatro municípios do Estado do Rio de Janeiro, 2005: resultados de inquérito domiciliar de base populacional. Ciênc Saúde Coletiva 2006; 11:643-55.

4. Barros MBA, Francisco PMSB, Zanchetta LM, Cesar CLG. Tendências das desigualdades sociais e demográficas na prevalência de doenças crônicas no Brasil, PNAD: 2003-2008. Ciênc Saúde Coletiva 2011; 16:3755-68.

\section{Agradecimentos}

À FAPESP (Projeto de Políticas Públicas, processo no 88/14099) e à Secretaria Estadual de Saúde de São Paulo pelo financiamento do trabalho de campo do ISA-SP. Ao CNPq (processo no 409747/2006-8) pelo financiamento do trabalho de campo do ISA-Camp. À FAPESP pela bolsa de pós-doutorado de P. M. S. B. Francisco e ao CNPq pelas bolsas de produtividade de M. B. A. Barros, C. L. G. César e M. Goldbaun.
5. Moraes JC, Ribeiro MCSA. Desigualdades sociais e cobertura vicinal: uso de inquéritos domiciliares. Rev Bras Epidemiol 2008; 11 Suppl 1:S113-24.

6. Lima-Costa MF, Matos DL. Prevalência e fatores associados à realização da mamografia na faixa etária de 50-69 anos: um estudo baseado na Pesquisa Nacional por Amostra de Domicílios (2003). Cad Saúde Pública 2007; 23:1665-73.

7. Travassos C, Viacava F, Laguardia J. Os suplementos saúde na Pesquisa Nacional por Amostra de Domicílios (PNAD) no Brasil. Rev Bras Epidemiol 2008; 11 Suppl 1:98-112.

8. Wünsch Filho V, Mirra AP, López RVM, Antunes L. Tabagismo e câncer no Brasil: evidências e perspectivas. Rev Bras Epidemiol 2010; 13:175-87.

9. Barros MBA. Inquéritos domiciliares de saúde: potencialidades e desafios. Rev Bras Epidemiol 2008 11 Suppl 1:S6-19. 
10. Borrel C, Rodríguez-Sanz M. Aspectos metodológicos de las encuestas de salud por entrevista: aportaciones de la Encuesta de Salud de Barcelona 2006. Rev Bras Epidemiol 2008; 11 Suppl 1:46-57.

11. Galán I, Rodríguez-Artalejo F, Tobías A, Gandarillas A, Zorrilla B. Vigilancia de los factores de riesgo de las enfermedades no transmisibles mediante encuesta telefónica: resultados de la comunidad de Madrid en el período 1995-2003. Gac Sanit 2005; 19:193-205.

12. Viacava F. Informações em saúde: a importância dos inquéritos populacionais. Ciênc Saúde Coletiva $2002 ; 7: 607-21$

13. Malta DC, Cezário AC, Moura L, Moraes Neto OL, Silva Junior JB. A construção da vigilância e prevenção das doenças crônicas não transmissíveis no contexto do Sistema Único de Saúde. Epidemiol Serv Saúde 2006; 15:47-65.

14. Li C, Balluz LS, Ford ES, Okoro CA, Zhao G, Pierannunzi C. A comparison of prevalence estimates for selected health indicators and chronic diseases or conditions from the Behavioral Risk Factor Surveillance System, the National Health Interview Survey, and the National Health and Nutrition Examination Survey, 2007-2008. Prev Med 2012; 54:381-7.

15. Ministério da Saúde. VIGITEL Brasil 2006. Vigilância de fatores de risco e proteção para doenças crônicas por inquérito telefônico: estimativas sobre freqüência e distribuição sócio-demográfica de fatores de risco e proteção para doenças crônicas nas capitais dos 26 estados brasileiros e no Distrito Federal em 2006. Brasília: Ministério da Saúde; 2007. (Série G. Estatísticas e Informação em Saúde).

16. Lee S, Davis WW, Nguyen HA, McNeel TS, Brick JM, Flores-Cervantes I. Examining trends and averages using combined cross-sectional survey data from multiple years. CHIS Methodology Paper, 2007. http://www.chis.ucla.edu/pdf/paper_trends_ave rages.pdf (acessado em 19/Set/2010).

17. Cesar CLG. Metodologia. In: Cesar CLG, Carandina L, Alves MCGP, Barros MBA, Goldbaum M, organizadores. Saúde e condição de vida em São Paulo: inquérito multicêntrico de saúde no Estado de São Paulo. São Paulo: Faculdade de Saúde Pública, Universidade de São Paulo; 2005. p. 37-46.

18. Alves MCGP. Plano de amostragem. In: Cesar CLG, Carandina L, Alves MCGP, Barros MBA, Goldbaum M, organizadores. Saúde e condição de vida em São Paulo: inquérito multicêntrico de saúde no Estado de São Paulo. São Paulo: Faculdade de Saúde Pública, Universidade de São Paulo; 2005. p. 47-62.

19. Organización Mundial de la Salud. El estado físico: uso e interpretación de la antropometría. Genebra: Organización Mundial de la Salud; 1995. (Serie de Informes Técnicos, 854).

20. Pessoa DGC, Nascimento Silva PL. Análise de dados amostrais complexos. São Paulo: Associação Brasileira de Estatística; 1998.

21. Kish L. Survey sampling. New York: John Wiley and Sons; 1965.
22. Jardim PCBV, Gondim MRP, Monego ET, Moreira HG, Vitorino PVO, Souza WKSB, et al. Hipertensão arterial e alguns fatores de risco em uma capital brasileira. Arq Bras Cardiol 2007; 88:452-7.

23. Pinheiro RS, Viacava F, Travassos C, Brito AS. Gênero, morbidade, acesso e utilização de serviços de saúde no Brasil. Ciênc Saúde Coletiva 2002; 7:687-707.

24. Arrais PSP. Medicamentos: consumo e reações adversas: um estudo de base populacional. Fortaleza: Edições UFC; 2009.

25. Costa KS, Barros MBA, Francisco PMSB, Cesar CLG, Goldbaum M, Carandina L. Utilização de medicamentos e fatores associados: um estudo de base populacional no Município de Campinas, São Paulo, Brasil. Cad Saúde Pública 2011; 27:649-58.

26. Barros MBA, Zanchetta LM, Moura EC, Malta DC. Auto-avaliação da saúde e fatores associados, Brasil, 2006. Rev Saúde Pública 2009; 43 Suppl 2: S27-37.

27. Dachs JNW, Santos APR. Auto-avaliação do estado de saúde no Brasil: análise dos dados da PNAD/2003. Ciênc Saúde Coletiva 2006; 11:887-94.

28. Blazer DG. How do you feel about...? Health outcomes in late and self-perceptions of health and well-being. Gerontologist 2008; 48:415-22.

29. Quesnel-Vallé A. Self-rated health: caught in the crossfire of the quest for 'true' health? [Editorial]. Int J Epidemiol 2007; 36:1161-4.

30. Idler EL, Benyamini Y. Self-rated health and mortality: a review of twenty-seven community studies. J Health Soc Behav 1997; 38:21-37.

31. World Health Organization. Obesity: preventing and managing the global epidemic. Report of a WHO Consultation Group on Obesity. Geneva: World Health Organization; 1998.

32. Malta DC, Moura EC, Castro AM, Cruz DKA, Morais Neto OL, Monteiro CA. Padrão de atividade física em adultos brasileiros: resultados de um inquérito por entrevistas telefônicas, 2006. Epidemiol Serv Saúde 2009; 18:7-16.

33. Vogel T, Brechat PH, Leprete PM, Kaltenbach G, et al. Health benefits of physical activity in older patients: a review. Int J Clin Pract 2009; 63:303-20.

34. World Health Organization. WHO report on the global tobacco epidemic, 2009. http://www.who. int/tobacco/mpower/en/index.html (acessado em 03/Mar/2011).

35. Silva GA, Valente JG, Malta DC. Tendências do tabagismo na população adulta das capitais brasileiras: uma análise dos dados de inquéritos telefônicos de 2006 a 2009. Rev Bras Epidemiol 2011; 14 Suppl 1:S103-14.

36. Malta DC, Moura EC, Silva AS, Oliveira PPV, CostaSilva VJL. Prevalência do tabagismo em adultos residentes nas capitais dos estados e no Distrito Federal, Brasil 2008. J Bras Pneumol 2012; 36: 75-85.

37. Fernandes LS, Peres MA. Associação entre atenção básica em saúde bucal e indicadores socioeconômicos municipais. Rev Saúde Pública 2005; 39:930-6. 
38. Coordenação de Educação, Coordenação Geral de Ações Estratégicas, Instituto Nacional de Câncer José Alencar Gomes da Silva. ABC do câncer: abordagens básicas para o controle do câncer. 2a Ed. Rio de Janeiro: Instituto Nacional de Câncer José Alencar Gomes da Silva; 2012.

39. Segri NJ, Francisco PMSB, Alves MCGP, Barros MBA, Cesar CLG, Goldbaum M, et al. Práticas preventivas de detecção de câncer em mulheres: comparação das estimativas dos inquéritos de saúde (ISA - Capital) e vigilância de fatores de risco e proteção para doenças crônicas por inquérito telefônico (VIGITEL - São Paulo). Rev Bras Epidemiol 2011; 14 Suppl 1:31-43.

40. Viacava F, Souza-Junior PRB, Moreira RS. Estimativas da cobertura de mamografia segundo inquéritos de saúde no Brasil. Rev Saúde Pública 2009; 43 Suppl 2:S117-25.

41. Amorim VMSL, Barros MBA, Cesar CLG, Carandina L, Goldbaum M. Fatores associados a não realização da mamografia e do exame clínico das mamas: um estudo de base populacional em Campinas, São Paulo, Brasil. Cad Saúde Pública 2008; 24:2623-32.

42. Nichol KL, Nordin J, Mullooly J, Lask R, Filbrandt $\mathrm{K}$, Iwane $\mathrm{M}$. Influenza vaccination and reduction in hospitalizations for cardiac disease and stroke among the elderly. N Engl J Med 2003; 348:132232.

43. Gross PA, Hermogenes AW, Sacks HS, Lau J, Levandowski RA. The efficacy of influenza vaccine in elderly persons. A meta-analysis and review of the literature. Ann Intern Med 1995; 123:517-28.
44. Centro de Vigilância Epidemiológica Prof. Alexandre Vranjac, Secretaria de Estado de Saúde de São Paulo. Campanha nacional de vacinação para o idoso 2009. Deixe a gripe na saudade. Vacine-se. São Paulo: Secretaria de Estado de Saúde de São Paulo; 2009. (Informe Influenza, 2009).

45. Francisco PMSB, Barros MBA, Cordeiro MRD. Vacinação contra influenza em idosos: prevalência, fatores associados e motivos da não-adesão em Campinas, São Paulo, Brasil. Cad Saúde Pública 2011; 27:417-26.

46. Burns VE, Ring C, Carroll D. Factors influencing influenza vaccination uptake in an elderly, community-based sample. Vaccine 2005; 23:3604-8.

47. Ferreira AD, César CC, Malta DC, Andrade ACS, Ramos CGC, Proietti FA, et al. Validade de estimativas obtidas por inquérito telefônico: comparação entre VIGITEL 2008 e Inquérito Saúde em Beagá. Rev Bras Epidemiol 2011; 14 Suppl 1:S16-30.

48. Francisco PMSB, Barros MBA, Segri NJ, Cesar CL, Malta DA. Comparação de estimativas para o auto-relato de condições crônicas: ISACamp e Vigitel-Campinas. Rev Bras Epidemiol 2011; 14 Suppl 1:5-15.

49. Waldman EA, Novaes HMD, Albuquerque MFM, Latorre MRDO, Ribeiro MCSA, Vasconcellos M, et al. Inquéritos populacionais: aspectos metodológicos operacionais e éticos. Rev Bras Epidemiol 2008; 11 Suppl 1:168-79.

50. Rede Portuguesa de Cidades Saudáveis. Perfis de saúde das cidades: uma análise do progresso. Brasília: Organização Mundial da Saúde; 1998.

Recebido em 28/Set/2012

Vertsão final reapresentada em 23/Jan/2013

Aprovado em 06/Fev/2013 